\title{
FABRICAÇÃO DE UM FORNO DE TRATAMENTO TÉRMICO VISANDO OPORTUNIDADES DE PRESTAÇÃO DE SERVIÇOS PARA DESENVOLVIMENTO DE NOVOS MATERIAIS E PROCESSOS PARA A INDÚSTRIA METALÚRGICA, CERÂMICA, DE POLÍMEROS E DE MICROELETRÔNICA ${ }^{1}$
}

\author{
MANUFACTURING OF A THERMAL TREATMENT OVEN AIMING \\ OPPORTUNITIES FOR THE PROVISION OF SERVICES FOR THE \\ DEVELOPMENT OF NEW MATERIALS AND PROCESSES FOR THE \\ METALLURGICAL, CERAMIC, POLYMER AND MICROELECTRONIC INDUSTRY
}

\author{
Recebido: 30/08/2017 - Aprovado: 14/10/2017 - Publicado: 30/12/2017 \\ Processo de Avaliação: Double Blind Review
}

\author{
Gerson Cristian Marques da Silva ${ }^{2}$ \\ Tecnólogo em Manutenção Industrial \\ FATEC-Osasco \\ gjd_marques@hotmail.com
}

\author{
Irley de Souza Oliveira ${ }^{1}$ \\ Tecnólogo em Manutenção Industrial \\ FATEC-Osasco \\ irley-ig@hotmail.com \\ Vagner Rodrigues de Souza ${ }^{1}$ \\ Tecnólogo em Manutenção Industrial \\ FATEC-Osasco
}

\begin{abstract}
${ }^{1}$ Os autores deste projeto agradecem: Indústrias Brasileiras de Artigos Refratários IBAR Ltda - pela doação do concreto refratário e disponibilização do seu equipamento e funcionários para elaboração para envasamento dos moldes e cura do mesmo (contato: +55 114634.6600 ou e-mail: ibarvendas@ibar.com.br); Esclafarma Soldas Ltda - localizada no Jardim Amalia II (e-mail para contato: roan.retse@uol.com.br); Promantec engenharia e soluções eirele ME - pela elaboração dos sistemas elétrico do forno: potência, comando e controle - localizada no Jardim Amalia II (e-mail para contato: rodrigo@promantec.com.br); Germak comércio de resistência Ltda - Orientação em relação aos elementos de aquecimento do forno e fabricação das resistências apenas a custo de produção (telefone para contato: +55 11 56618267); Escola SENAI Nadir Dias de Figueiredo - pela elaboração dos moldes para confeccionar os refratários da câmara do forno e doação do material usado e disponibilização de sua infraestrutura (telefone para contato: +55 11 3685-7999); Fyn Soluções - pela disponibilização de funcionário, infraestrutura, material estrutural e alumínio usado nos experimentos, contribuindo para a realização do projeto (telefone para contato: 55114384 4746); Também agradecemos ao professor Eng. Carlos Freitas da disciplina de Trabalho de Graduação pelo apoio e auxílio no controle de cronogramas deste trabalho. Ao Sr. Paulo Rosa, auxiliar técnico da Fatec-Osasco que ajudou na realização de usinagem, para confecção dos moldes para o envase do concreto refratário. Ao Sr. João Carlos Sartori pela doação do controlador CONTEMP e do TERMOPAR que foram essenciais para o projeto. Ao professor MSc. Carlos Sartori pelas suas discussões sobre o processo de fundição de metais e discussões sobre a ciência dos materiais. Ao professor MSc. Antônio Carlos de Arruda por ter doado R\$ 1.140,00 para a compra do tubo de Quartzo com pureza de 99,99\% e pelas orientações e conversas. Ao professor MSc. Raphael Garcia Moreira pela doação de R\$1.140,00 relacionados à compra do tubo de Quartzo com pureza de $99,99 \%$, $\mathrm{R} \$ 111,98$ para compra de materiais elétricos e pela doação de cadinhos em Carbeto de Silício importados, por ter atuado como nosso orientador do projeto com foco em aplicações de alta tecnologia agregada e pelas revisões e discussões sobre o presente trabalho.
\end{abstract}

2Autor para correspondência: Faculdade de Tecnologia do Estado de São Paulo, Rua Pedro Rissato, 30, Vila dos Remédios, Osasco - SP, Brasil - CEP 06296-220.

REMIPE- Revista de Micro e Pequenas Empresas e Empreendedorismo da Fatec Osasco V. 3, N², jul.-dez. 2017. 
vagnervrs@hotmail.com

Kennedy Yoshinori de Souza

Tecnólogo em Manutenção Industrial

FATEC - Osasco

kennedy.yamashita1@gmail.com

Emerson Costa Santos

Engenheiro Mecânico

Instrutor na Escola SENAI Nadir Dias de Figueiredo

emerson.csantos@sp.senai.br

André Rosa Ferreira

Mestre em Engenharia Elétrica

Professor da FATEC - Osasco

andre.rferreira@fatec.sp.gov.br

Deocleciano Reis Martins

Graduado em Tecnologia Mecânica-Projetos

Professor da FATEC - Osasco

deocleciano.rmartins@fatec.sp.gov.br

RESUMO: Existem grandes oportunidades de negócios relacionados ao desenvolvimento de tratamento térmico para novos materiais e processos industriais, entre eles são destacados: metalurgia, cerâmica, polímeros e de microeletrônica. Observa-se que estas oportunidades nem sempre são sendo totalmente exploradas. Em geral a faixa de temperatura que promove tratamentos térmicos adequados para as indústrias alvo deste trabalho varia desde 50 até $1800{ }^{\circ} \mathrm{C}$ com diferentes tempos de residência. Fornos que sigam especificações de rampa de aquecimento e resfriamento, bem como grau de pureza para os processos da microeletrônica são encontrados apenas em grandes centros de pesquisa no Brasil. Por este motivo, o objetivo do artigo consiste no desenvolvimento de um forno visando atender demandas de prestação de serviço de pesquisa e desenvolvimento de novos processos e materiais. O projeto foi concluído a um custo de $\mathrm{R} \$ 11.997,90$, e apresentou aderência com a necessidade de micro e pequenas empresas que possam se beneficiar deste mercado. Como teste conceitual foi executado a fundição de liga de alumínio tipo 1200 H14.

Palavras-chave: Tratamento térmico, forno, microeletrônica, metalurgia, polímeros, cerâmica. 
ABSTRACT: There are great business opportunities related to the development of heat treatment for new materials and industrial processes, among which are outstanding: metallurgy, ceramics, polymers and microelectronics, these opportunities are not being explored. In general, the temperature range that promotes suitable heat treatments for the industries targeted for this work varies from 50 to $1800^{\circ} \mathrm{C}$ with different residence times. Ovens that follow heating and cooling ramp specifications as well as degree of purity for microelectronic processes are found only at major research centers in Brazil. In this work it was proposed by the manufacture of a thermal treatment oven to meet the demands of providing research service and development of new processes and materials. The project was completed at a cost of $R \$ 11,997.90$, and showed adherence to the need for micro and small companies that could be benefit from this market. As a conceptual test, the casting of 1200 H14 aluminum alloy was performed.

Keywords Water: heat treatment, oven; microelectronics; metallurgy; polymers; ceramics.

\section{INTRODUÇÃO}

Nos centros e em empresas da área de pesquisa e desenvolvimento no Brasil há número relevante de projetos para o desenvolvimento de novos materiais, que necessitam de tratamentos térmicos especializados (LASTRES; 2016). Entre os projetos desenvolvidos no país, recebem destaque os novos polímeros e cerâmicas, que promovem oportunidade de crescimento nacional no mercado internacional (AZEVEDO; STRECKER; 2017).

Estudos feitos nas regiões de Carapicuíba, Osasco, Taboão da Serra, Cotia e Embu, por exemplo, destacam ser uma grande oportunidade o desenvolvimento de novos materiais cerâmicos, a partir de resíduos provenientes do saneamento básico (CATOLICO; CARVALHO; JARQUE; 2015). Tais materiais são sujeitos a várias etapas de tratamentos térmicos.

Observa-se, também, a constante demanda por estudos na área de metalurgia (XAVIER; PLAUT; SCHÖN; 2014) bem como aqueles que envolvem o tratamento térmico como meio para melhorar as características de aços e suas aplicações (LAM; 2015). Outro importante setor metalúrgico em constante evolução é o setor do aço inoxidável (AGUIAR et al.; 2015). 
Por outro lado, entre as mais notáveis aplicações que emprega o tratamento térmico, a Tecnologia Metal-Oxide-Semiconductor (MOS) é a mais utilizada pelas indústrias, ambientes domésticos, calculadoras, computadores, equipamentos eletrônicos e, mais especificamente, nos smartphones, notebooks e eletrônicos de alta tecnologia. Esta tecnologia faz parte do transistor que é o componente básico dos processadores, memórias ou circuitos eletrônicos integrados (KIKUCHI; KITA; 2014). O processo de oxidação de uma lâmina de silício visando a construção das mais notáveis tecnologias atuais necessita de várias etapas de processamento térmico adequado (SUZUKI; 1982).

A pesquisa justifica-se, pois o domínio da tecnologia de tratamentos térmicos é economicamente atrativo, no Brasil houve somente um caso de liderança nacional e internacional, que é o caso da GERDAU (BITTENCOURT; NOVA; CORNACCHIONE; 2017). A siderurgia nacional demanda tratamentos térmicos em praticamente todas as etapas, logo possui uma expressiva participação no Produto Interno Bruto (PIB) brasileiro. Sendo que O PIB setorial no ano de 2010 foi de US\$ 45,7 bilhões, considerando de $2 \%$ do PIB nacional e $8,1 \%$ do PIB industrial em 2013. O saldo comercial do setor registra US\$ 3,9 bilhões que representou 13\% do saldo comercial do país em 2013 (ALBUQUERQUE; SILVA; MALUF; 2014).

O pouco domínio das etapas de tratamento térmico com grau de pureza consoante às aplicações de microeletrônica, ou seja, no processamento de silício visando produção de memórias, processadores e circuitos integrados leva o Brasil a perder um mercado muito valioso. O Brasil atualmente exporta silício metalúrgico (silício processado a temperaturas da ordem de $1000^{\circ} \mathrm{C}$ ) a um preço de US $\$ 2 / \mathrm{kg}$. Por outro lado, depois do silício metalúrgico ser manufaturado pelos países que dominam as demais etapas de fabricação, o preço das commodities chegam a varias de US\$ 50/kg até US\$ 1000/kg. Observa-se que muitas etapas de manufatura já são dominadas pela academia brasileira, porém, falta ainda difusão de conhecimento para as indústrias, bem como o investimento. (BREJÃO; MORAIS; VENDRAMETTO; 2014).

O Brasil possui inúmeros campos de aplicação para fornos industriais aplicados ao tratamento térmico de alta tecnologia, o valor agregado é elevado, contudo, nem sempre parece uma tarefa fácil fabricar ou adquirir um forno com tais especificações. Logo, este trabalho é justificado baseado no fato de que a oportunidade existe, sendo real para o setor 
de fabricação de fornos de tratamento térmico, visando serviços de desenvolvimento de novos materiais e processos para a indústria metalúrgica, cerâmica, de polímeros e de microeletrônica.

Deve ser realçado que entre as literaturas citadas, todos possuem ou pesquisador vinculado a instituições de Osasco, empresa de Osasco ou materiais provenientes da cidade de Osasco, o que sugere que este é um setor de oportunidades para Micro e Pequenas Empresas desta região em que foi desenvolvida a pesquisa.

O objetivo geral deste trabalho consiste em realizar a fabricação de fornos de tratamento térmico visando serviços de desenvolvimento de novos materiais e processos para a indústria metalúrgica, cerâmica, de polímeros e de microeletrônica de baixo custo, apropriado para os recursos disponíveis no município de Osasco do Estado de São Paulo.

Os objetivos específicos são:

- Fabricar um forno visando tratamentos térmicos para a indústria metalúrgica, cerâmica, de polímeros e de microeletrônica com baixo custo visando ser usado em laboratórios de pesquisa e desenvolvimento de Micro e Pequenas Empresas;

- Atender com grau de pureza interna aplicações em microcomponentes com escala de até $100 \mu \mathrm{m}$;

- Fundir alumínio puro em cadinho de carbeto de silício, visando fazer uma prova de funcionamento do forno fabricado;

- Identificar os custos da fabricação;

\section{FUNDAMENTAÇÃO TEÓRICA}

A cooperação entre indústria e academia visando o desenvolvimento nacional, ainda, é bastante prematura no Brasil, isso tem sido constatado em vários setores industriais. Entre os setores, destacam-se os que são objeto desta pesquisa: metalurgia, cerâmica, polímeros e microeletrônica (sendo esta última a de menor desenvolvimento nacional). Somente recentemente, por meio de estímulos de governo, é que há maior intensidade de desenvolvimento de tecnologias promovida por parcerias estratégicas entre indústria e academia (SILVA; FURTADO; VONORTAS; 2017). Estas parcerias passam pela 
cooperação na aquisição de sistemas de tratamento térmico de alta tecnologia, bem como a troca de conhecimentos técnicos na área (SILVA; FURTADO; VONORTAS; 2017).

A faixa de temperatura que promove tratamentos térmicos adequados para as indústrias alvo deste trabalho varia desde 50 até $1800{ }^{\circ} \mathrm{C}$ com diferentes tempos de residência (HERBST, Sebastian et al., 2016). Outro aspecto bastante relevante é o grau de pureza do tratamento térmico que deve ser compatível com o objetivo do tratamento térmico (BIRAT, Jean-Pierre, 2016).

No caso da indústria microeletrônica que visa a produção de componentes na escala de nanômetros até micrometros, essa especificação, ou seja, a necessidade de pureza extrema é um dos principais gargalos tecnológicos. Logo, uma contaminação com frações da ordem de 1 ppm (partes por milhão) de carbono, sódio, potássio e outros; podem inviabilizar processos de fabricação na etapa térmica, pois tal contaminação poderá migrar para camadas internas de microdispositivos promovendo alterações de funcionamento irreversíveis e não controladas (AITKEN et al.; 2013). Para atender a este mercado, um forno, além da necessidade de contar com equipamentos e processos de fabricação de alto grau de pureza, precisam ainda atingir faixas de temperatura da ordem de 900 a $1500{ }^{\circ} \mathrm{C}$ (SOMETANI; 2017).

No caso da indústria de polímeros, a faixa de 100 a $1200{ }^{\circ} \mathrm{C}$ é bem comum de ser encontrada nos processos de produção de polímeros (SATILMIS; BUDD; 2017). Esta faixa é compatível também com a indústria de cerâmicas (ZHANG et al.; 2014).

\section{MATERIAIS E MÉTODOS}

\subsection{LEVANTAMENTO DOS PRINCIPAIS MATERIAIS PARA FABRICAÇÃO DE UM FORNO E RESPECTIVOS PREÇOS}

Como metodologia de planejamento, foram inicialmente pesquisados fornecedores de materiais da região de Osasco, contudo, alguns materiais foram adquiridos de outras regiões, segue Tabela 1: 
Tabela 1. Lista de materiais e preços para fabricação do forno conforme especificações.

\begin{tabular}{|c|c|c|}
\hline Nome & Modelo Comercial/ Dados Técnicos & Preço \\
\hline Concreto refratário & Dados disponível no PDF "Concreto refratário (castibar_plw_41_ug)" & $R \$ 2.500,00$ \\
\hline \multicolumn{2}{|l|}{ Tubo de Quazto } & $R \$ 1.500,00$ \\
\hline Resistências elétrica & $\begin{array}{l}\text { Resistências confeccionadas em Kanthal A1 1,45, equivalente a } 0,2685 \text { Ohms por metro. } \\
\text { São } 12 \text { resistências instaladas de maneira distribuida ao redor da câmara dissipando } \\
\text { uma carga de } 8 \mathrm{KW}(220 \mathrm{~V} / 40 \mathrm{~A}) \text {. }\end{array}$ & $\mathrm{R} \$ 700,00$ \\
\hline Fibra Cerâmica & Dados disponivel no PDF "Manta Cerâmica (MT 64 - 12\%2c7 HPS)" & $R \$ 3.000,00$ \\
\hline Controlador & Dados em Anexo (Manual do Controlador) & $\mathrm{R} \$ 400,00$ \\
\hline Termopar & $\begin{array}{l}\text { Termopar com isolação mineral Tipo "K" com haste de } 6 \mathrm{~mm} \text { de diâmetro AISI } 310 \text { com } \\
\text { cabeçote KNC sem rosca de processo, comprimento útil }=400 \mathrm{~mm} \text {, temperatura de uso } \\
0+1100^{\circ} \mathrm{C} \text { (suporta picos de curta duração de } 1200^{\circ} \mathrm{C} \text { ) }\end{array}$ & $R \$ 160,00$ \\
\hline Chaparia externa aço carbono & Chapa de aço carbono $3000 \times 1000 \mathrm{~mm} \# 1,5 \mathrm{~mm}$ ( 2 unids) & $\mathrm{R} \$ 898,40$ \\
\hline Tubo Retângular estrutura da base & Tubo retangular $30 \times 50 \times 1,2 \mathrm{~mm}$ ( 5 barras de $6 \mathrm{mts}$ ) & $R \$ 275,50$ \\
\hline \multicolumn{2}{|c|}{ Berço do conjunto da câmara (Aço Inoxidável 31 Chapa de aço inoxidável 3102000 × 1000 × 6mm } & $\mathrm{R} \$ 1.872,00$ \\
\hline Alimentação das Resistências & $\begin{array}{l}\text { Utilizado cabo elétrico flexível de } 6 \mathrm{~mm}^{2} \text { e terminais olhais de } 8 \mathrm{~mm} \text { de diâmetro para } \\
\text { conectar os cabos aos elétrodos de aço inoxidável } 310 \text { / } 8 \mathrm{~mm} \text { de diâmetro das } \\
\text { resistências elétricas. }\end{array}$ & $R \$ 180,00$ \\
\hline Tubo cerâmico das resistências & $\begin{array}{l}\text { Turbo cerâmico confeccionado por meio de granulometria fina, composto de } 99,8 \% \text { de } \\
\text { aluminia, tornando excelentes isoladores, resistentes a altas temperaturas, corrosão e } \\
\text { choque térmico. Formato do tubo usado: Diâmetro externo } 17 \mathrm{~mm} \text {, Diâmetro interno de } \\
11 \mathrm{~mm} \text { e comprimento de } 400 \mathrm{~mm} \text {. }\end{array}$ & $R \$ 132,00$ \\
\hline Tubo com Frange & $\begin{array}{l}\text { Turbo com frange cerâmico confeccionado por meio de granulometria fina, composto de } \\
99,8 \% \text { de aluminia, tornando excelentes isoladores, resistentes a altas temperaturas, } \\
\text { corrosão e choque térmico. }\end{array}$ & $R \$ 42,00$ \\
\hline Material / componentes elétricos & Coontatores, disjuntores, cabo, painel elétrico etc. & $\mathrm{R} \$ 338,00$ \\
\hline
\end{tabular}

Fonte: Elaborado pelos autores, 2017.

O projeto foi iniciado com um investimento estimado de R\$11.997,90. É digno de nota que o concreto refratário, resistências elétricas e fibra cerâmica correspondem juntos a $55 \%$ do custo de materiais, por outro lado, o tubo de quartzo com pureza de 99,99\% representa $13 \%$ do custo total do forno, este tubo terá sua maior importância em aplicações onde a pureza seja extremamente necessária.

\subsection{PROJETO GERAL}

O projeto do forno foi realizado com uma licença acadêmica do SolidWorks. As figuras Figura 1, Figura 2 e Figura 3 mostram as principais dimensões, materiais e suas posições no projeto, conforme seguem: 
Figura 1 - Vista do projeto 2D do forno. Medidas em milímetros.
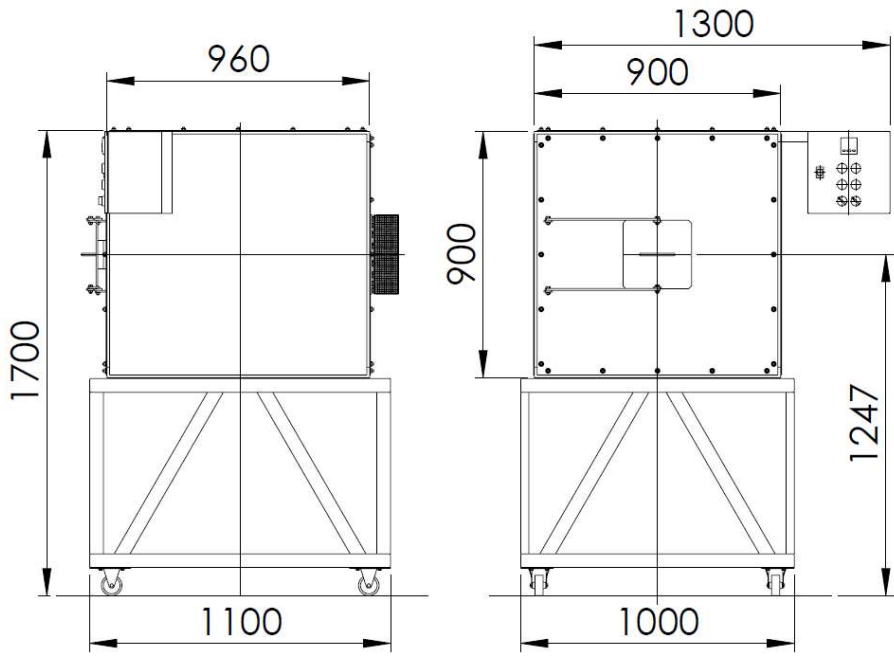

Fonte: Elaborado pelos autores, 2017.

Figura 2 - Vista em corte do forno mostrando as principais partes e peças.

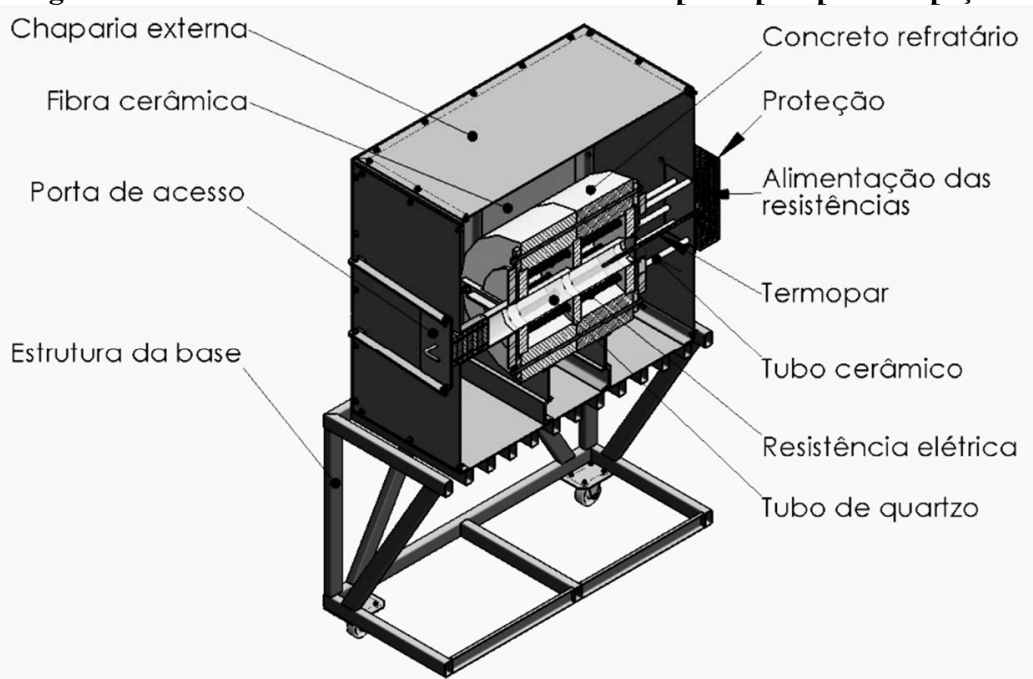

Fonte: acervo pessoal, 2017. 
Figura 3 - Vista geral do projeto do forno.

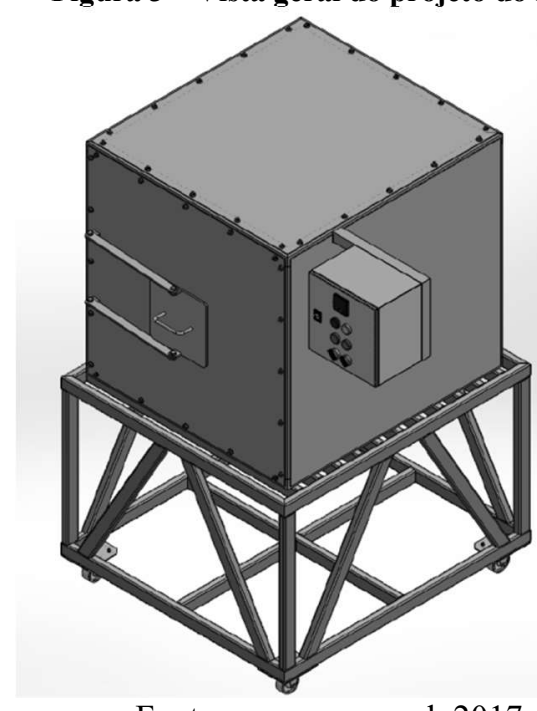

Fonte: acervo pessoal, 2017.

A potência do forno foi dimensionada para $8,0 \mathrm{~kW}$ e confeccionadas com resistências tipo KANTHAL $\mathrm{A}^{3}$ permitindo atingir temperaturas de até $1.200^{\circ} \mathrm{C}$.

\subsection{ETAPAS DE FABRICAÇÃO}

Nas figuras que seguem, são mostradas as etapas de fabricação do forno. Deve ser observado que todas as etapas são compatíveis com equipamentos de fácil acesso, podendo ser este projeto realizado por oficinas vinculadas a Micro e Pequenas empresas. A Figura 4 apresenta o processo de soldagem da estrutura externa:

\footnotetext{
${ }^{3}$ Kanthal A-1 é uma liga de ferro-cromo-alumínio para aplicações de temperaturas da ordem de $1400^{\circ} \mathrm{C}$. A liga é caracterizada por alta resistividade e resistência à oxidação.
} 
Figura 4. Soldagem das estruturas externas do forno usando processo MIG/MAG.

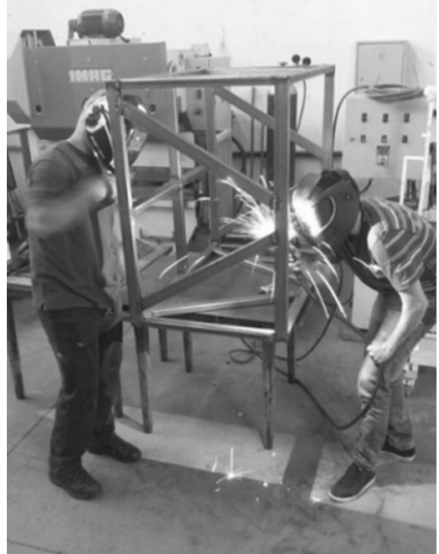

Fonte: acervo pessoal, 2017.

A Figura 5 mostra o delicado processo de montagem dos refratários internos e processo de isolação com a fibra isolante apropriada para altas temperaturas:

Figura 5. Detalhes das estruturas internas com proteção para temperaturas de até $1600^{\circ} \mathrm{C}$. A) Refratários especiais para suporte das resistências. B) Isolantes de fibra cerâmica para altas temperaturas.

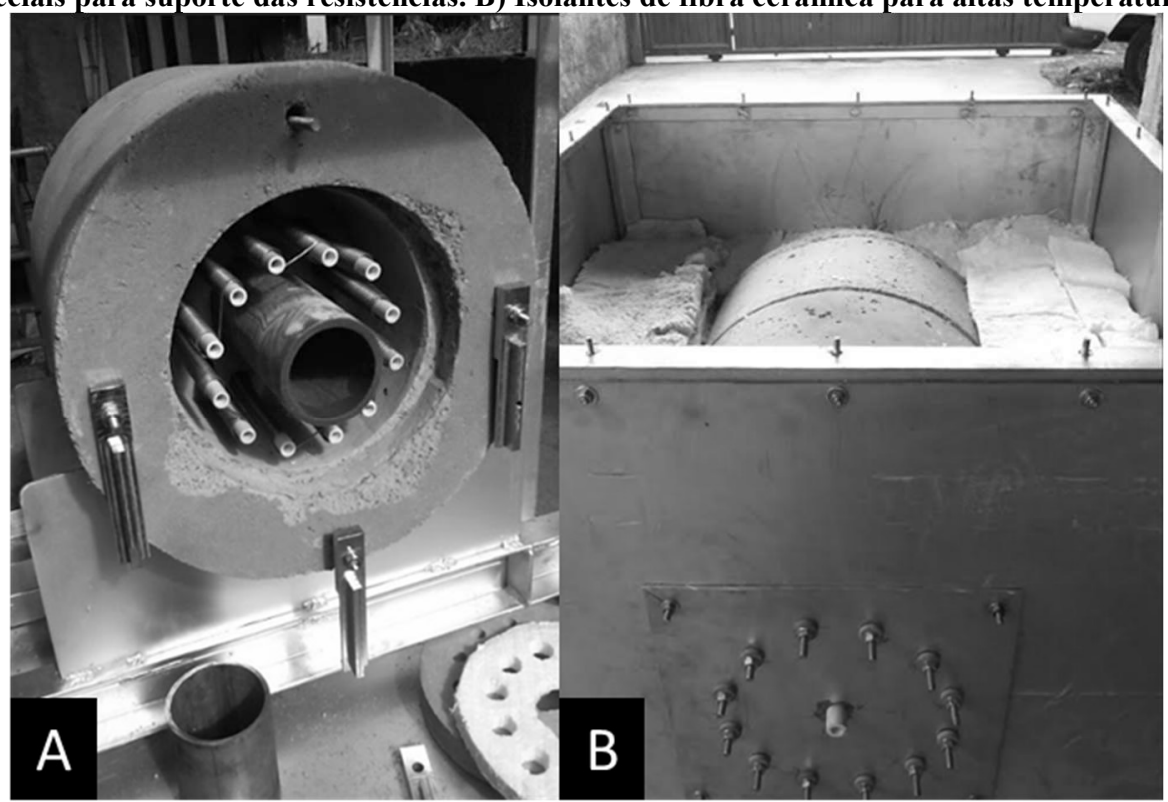

Fonte: acervo pessoal, 2017.

A Figura 6 mostra o resultado final da confecção manual das resistências que providenciam potência de $8 \mathrm{~kW}$ ao forno: 
Figura 6. Resultado da preparação manual das resistências em Kanthal A1.

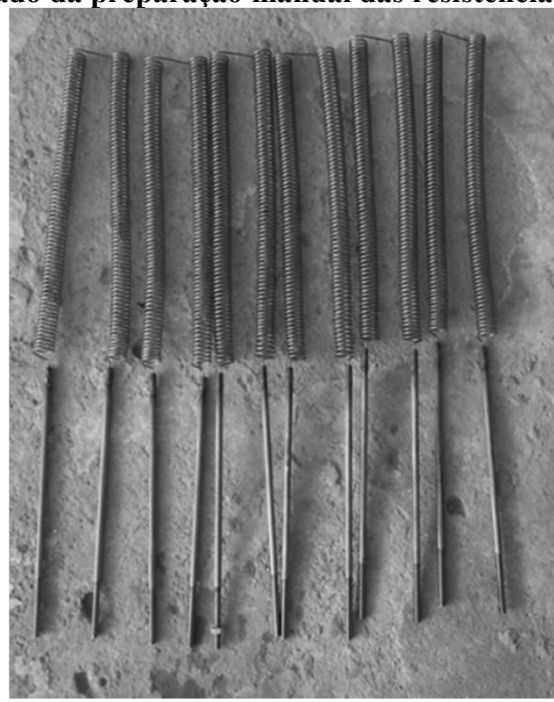

Fonte: acervo pessoal, 2017.

A Figura 7 mostra a etapa de construção do comando elétrico de acionamento e controle das resistências do forno e sistema de proteção:

Figura 7. Etapas de montagem do circuito elétrico de acionamento e controle.

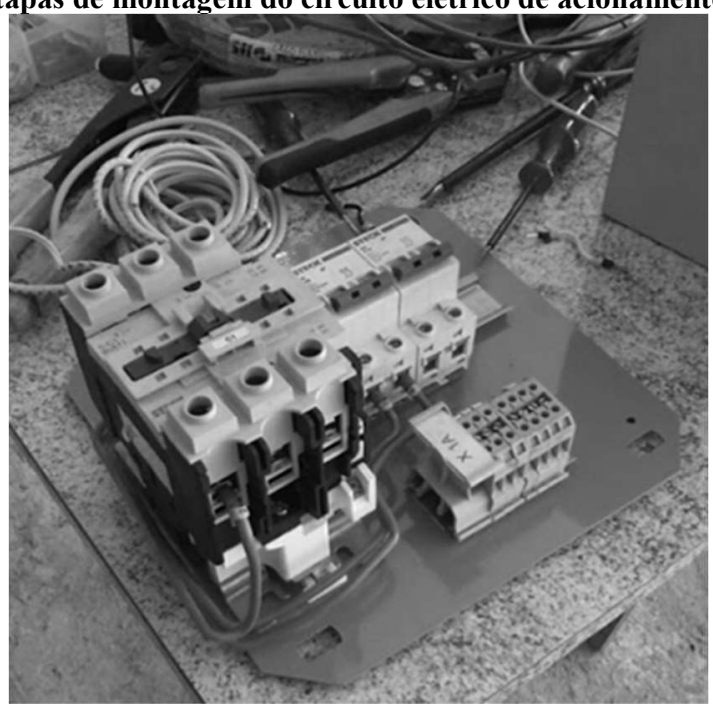

Fonte: acervo pessoal, 2017.

\section{APRESENTAÇÃO E ANALISE DE RESULTADOS}

A fabricação de um forno de tratamento térmico visando prestação de serviços de desenvolvimento de novos materiais e processos para a indústria metalúrgica, cerâmica, de polímeros e de eletrônica foi concluída com sucesso conforme Figura 8: 
Figura 8. Vista do forno totalmente concluído e operacional.

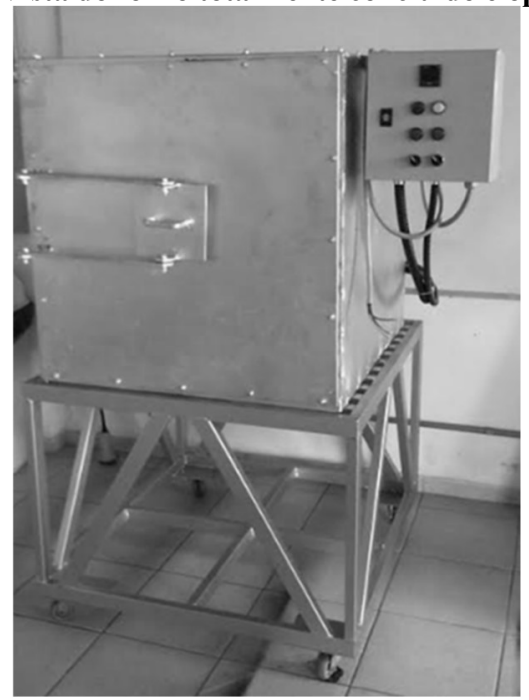

Fonte: acervo pessoal, 2017.

Um primeiro teste inicial visando fundir alumínio foi realizado com sucesso. Este teste teve a finalidade de caracterizar os principais parâmetros do forno, tais como: rampa de aquecimento, resfriamento, características de operacionalidade, eficiência da isolação térmica visando segurança dos operadores e demais aspectos técnicos, conforme a Figura 9:

Figura 9. A) Vista do forno em operação, aquecido a $1000^{\circ} \mathrm{C}$. B) Alumínio fundido pelo forno.

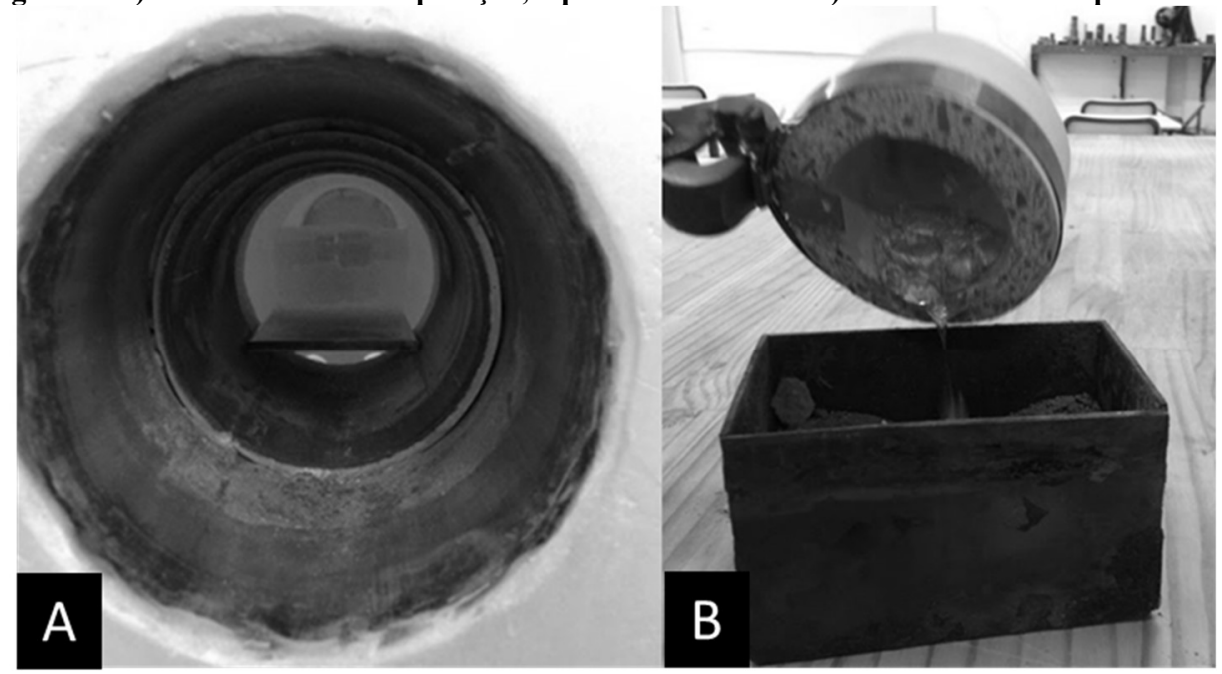

Fonte: acervo pessoal, 2017.

O forno fabricado apresentou bom funcionamento, e bons aspectos de operacionalidade e segurança. O custo total de fabricação foi de R $\$ 11.997,90$, este é um valor que pode ser alcançado pela micro ou pequena empresa por meio de programas como, 
por exemplo, o Desenvolve SP - Agência de Desenvolvimento Paulista (www.desenvolvesp.com.br), o preço médio da energia em São Paulo é de R\$ 0,34873/kWh, o que também deverá ser levado em consideração durante a precificação dos serviços especializados a serem prestados por meio deste forno.

Isso significa que com um forno desses, a micro ou pequena empresa pode oferecer serviços de tratamento térmico para finalidade de desenvolvimento de materiais e processos com alto valor agregado tendo como principal custo a mão de obra que gira em torno de 3 a 5 salários mínimos em São Paulo (BALTAR; 2016) somado ao custo da energia, por outro lado, o valor agregado em serviços especializados é bastante alto, contudo, não foram encontrados na literatura índices que permitam estimar o valor da hora de tratamento térmicos oferecidos por fornos de alta tecnologia como este, contudo, empresários interessados neste serviço mencionam ser um valor de $\mathrm{R} \$ 550,00 /$ hora bastante conveniente tanto para o prestador de serviço especializado quanto para o contratante do serviço.

\section{CONSIDERAÇÕES FINAIS}

Um forno de tratamento térmico visando serviços de desenvolvimento de novos materiais e processos para a indústria metalúrgica, cerâmica, de polímeros e de microeletrônica foi concluído e testado. Como teste inicial uma liga de alumínio foi fundida com sucesso.

$\mathrm{O}$ projeto se mostrou bastante competitivo e eficiente em termos de custo e operacionalidade, por outro lado, não há índices históricos desse tipo de prestação de serviços o que sugere que este é um novo mercado a ser explorado ou um mercado reprimido.

O mercado relacionado ao desenvolvimento de materiais e processos tecnológicos das áreas de metalurgia, cerâmica, de polímeros e microeletrônica pode ser fortemente impulsionado no Brasil se este tipo de serviço atingir uma massa crítica mínima, ou seja, um número mínimo de empresas que possam oferecer isso a um preço de mercado acessível a todos os interessados. 
Como perspectivas futuras são sugeridas para o presente trabalho as seguintes atividades:

- Desenvolvimento de matérias cerâmicas em parceria com empresas deste setor;

- Desenvolvimento de ligas metálicas de alta tecnologia com empresas do setor;

- Desenvolvimento de novos processos de tratamento de polímeros com empresas deste setor;

- Desenvolvimento de etapas de microeletrônica com institutos ou empresas vinculados ao setor de pesquisa e desenvolvimento tecnológico;

- Aferição do preço da hora de tratamentos térmicos especializados por setor;

- Ajustes tecnológicos do forno em relação à atual versão.

\section{REFERÊNCIAS}

AGUIAR, Isabela Viegas et al. Microstructure characterization of a duplex stainless steel weld by electron backscattering diffraction and orientation imaging microscopy techniques. Matéria (Rio de Janeiro), v. 20, n. 1, p. 212-226, 2015.

AITKEN, JOHN M.; IRENE, EUGENE A. Silicon Dioxide Films in Semiconductor Devices. Glass IV: Treatise on Materials Science and Technology, v. 26, 2013.

ALBUQUERQUE, Pedro HM; SILVA, Letícia da Costa; MALUF, Yuri Sampaio. Estimating the influence of macroeconomic variables on steel sales organizations using ARMAX. Gestão \& Produção, v. 21, n. 3, p. 648-659, 2014.

BIRAT, Jean-Pierre. Steel cleanliness and environmental metallurgy. Metallurgical Research \& Technology, v. 113, n. 2, p. 201, 2016.

BITTENCOURT, João Paulo; NOVA, Silvia Pereira de Castro Casa; CORNACCHIONE, Edgard. Gerdau's Men of Steel: A Unique Case of Leadership Development in Brazil. In: Leadership Development in Emerging Market Economies. Palgrave Macmillan US, 2017. p. 15-35.

BREJÃO, Antônio Sérgio; DE OLIVEIRA MORAIS, Marcos; VENDRAMETTO, Oduvaldo. A Macro Sectorial Study of Semiconductor Production. In: IFIP International Conference on Advances in Production Management Systems. Springer Berlin Heidelberg, 2014. p. 513-520.

CATOLICO, Ana Carolina Chaves; CARVALHO, Luciana Crivelare Gomes; JARQUE, Natalia Alegreiro. APROVEITAMENTO DE RESÍDUOS DO SANEAMENTO BÁSICO NA FABRICAÇÃO DE CERÂMICA VERMELHA. Periódico Eletrônico Fórum Ambiental da Alta Paulista, v. 11, n. 9, 2015. 
DE ANDRADE BALTAR, Paulo Eduardo. Rotatividade da mão-de-obra e diferenciação das remunerações no Estado de São Paulo. Economia e Sociedade, v. 3, n. 1, p. 99-113, 2016.

DE MORAES SILVA, Diego R.; FURTADO, André T.; VONORTAS, Nicholas S. University-industry R\&D cooperation in Brazil: a sectoral approach. The Journal of Technology Transfer, p. 1-31, 2017.

DE SOUZA AZEVEDO, Adriano Galvão; STRECKER, Kurt. BRAZILIAN FLY ASH BASED INORGANIC POLYMERS PRODUCTION USING DIFFERENT ALKALI ACTIVATOR SOLUTIONS. Ceramics International, 2017.

HERBST, Sebastian et al. Process Integrated Heat Treatment of a Microalloyed Medium Carbon Steel: Microstructure and Mechanical Properties. Journal of Materials Engineering and Performance, v. 25, n. 4, p. 1453-1462, 2016.

KIKUCHI, Richard Heihachiro; KITA, Koji. Fabrication of SiO2/4H-SiC (0001) interface with nearly ideal capacitance-voltage characteristics by thermal oxidation. Applied Physics Letters, v. 105, n. 3, p. 032106, 2014.

LAM, Kelvin. Análise microestrutural e caracterização mecânica do aço AISI 4350 submetido a diferentes tratamentos térmicos. 2015.

LASTRES, Helena M. AM. Effects on Third World Development. New Generic Technologies in Developing Countries, p. 68, 2016.

SATILMIS, Bekir; BUDD, Peter M. Selective dye adsorption by chemically-modified and thermally-treated polymers of intrinsic microporosity. Journal of Colloid and Interface Science, v. 492, p. 81-91, 2017.

SOMETANI, Mitsuru et al. Impact of rapid cooling process in ultrahigh-temperature oxidation of 4H-SiC (0001). Japanese Journal of Applied Physics, v. 56, n. 4S, p. 04CR04, 2017.

SUZUKI, Akira et al. Thermal Oxidation of SiC and Electrical Properties of Al-SiO2-SiC MOS Structure. Japanese Journal of Applied Physics, v. 21, n. 4R, p. 579, 1982.

XAVIER, Marcos Domingos; PLAUT, Ronald Lesley; SCHÖN, Cláudio Geraldo. Uniaxial near plane strain tensile tests applied to the determination of the FLC0 formabillity parameter. Materials Research, v. 17, n. 4, p. 982-986, 2014.

ZHANG, Li-yuan et al. Preparation of high open porosity ceramic foams via direct foaming molded and dried at room temperature. Journal of the European Ceramic Society, v. 34, n. 10, p. 2443-2452, 2014. 